\title{
The X-ray Structure and Spectrum of the Pulsar Wind Nebula Surrounding PSR B1853+01 in W44
}

\author{
R. Petre, K. D. Kuntz ${ }^{1}$ \\ NASA Goddard Space Flight Center, Greenbelt, MD 20771 \\ and
}

R. L. Shelton

The Henry A. Rowland Department of Physics and Astronomy, Johns Hopkins University, 3400 North

Charles Street, Baltimore, MD 21218

\begin{abstract}
We present the result of a Chandra ACIS observation of the pulsar PSR B1853+01 and its associated pulsar wind nebula (PWN), embedded within the supernova remnant W44. A hard band ACIS map cleanly distinguishes the PWN from the thermal emission of W44. The nebula is extended in the north-south direction, with an extent about half that of the radio emission. Morphological differences between the X-ray and radio images are apparent. Spectral fitting reveals a clear difference in spectral index between the hard emission from PSR B1853+01 ( $\Gamma \sim$ 1.4 ) and the extended nebula $(\Gamma \sim 2.2)$. The more accurate values for the $X$-ray flux and spectral index are used refine estimates for PWN parameters, including magnetic field strength, the average Lorentz factor $\gamma$ of the particles in the wind, the magnetization parameter $\sigma$, and the ratio $\mathrm{k}$ of electrons to other particles.
\end{abstract}

Subject headings: supernova remnants--ISM:individual(W 44)-X-rays:general

\section{Introduction}

The remarkable pulsar wind nebula associated with PSR B1853+01 and embedded in the evolved, mixed-morphology supernova remnant W44 is of particular interest for several reasons. It is the oldest known pulsar wind nebula (PWN), associated with an active pulsar (only the PWN in IC 443 is thought to be older - Bocchino \& Bychkov 2001). Its age of $20,000 \mathrm{yr}$ is estimated from the PSR B1853+01 spindown (Wolszczan, Cordes \& Dewey 1991). At the same time, PSR B1853+01 is one of the 10 youngest known pulsars. Thus the nebula may allow the testing of hypotheses regarding PWN evolution, and serves as a bridge between the young, active pulsars in supernova remnants and the preponderant population of iso-

\footnotetext{
${ }^{1}$ Department of Physics, University of Maryland Baltimore County, 1000 Hilltop Circle, Baltimore MD 21250
}

lated, old radio pulsars. Additionally, because of its high proper motion, the pulsar leaves a record of its evolution embedded in the extended nebula. A combination of high resolution radio and $\mathrm{X}$-ray observations can potentially disentangle this record. For example, radio measurements of the extent of the nebula perpendicular to the direction of motion provide means for setting an upper limit to the lifetime of the radio emitting electrons $(\sim 6,000 \mathrm{yr})$ unavailable from observation of wind nebulae associated with stationary pulsars. Finally, some of the properties of this PWN are similar enough to those of the much younger and more luminous Crab Nebula (Chevalier 2000) to invite comparisons and speculation regarding the reason for the similarities.

With the clearest view of the X-ray universe, especially above $3 \mathrm{keV}$, now available via the Chandra X-ray Observatory, more comprehensive stud- 
ies of embedded PWNs become feasible. In this paper we use Chandra's Advanced CCD Imaging Spectrometer (ACIS) to reveal the $\mathrm{X}$-ray structure and spectrum of the synchrotron nebula surrounding the W44 pulsar, and provide a more careful look at the $\mathrm{X}$-ray spectrum and its spatial variation. As has been shown in numerous other works (e.g., Harrus et al. 1996; Frail et al. 1996; Torii et al. 2000), the study of the wind nebulae surrounding pulsars provides a means for understanding the energetics of pulsars, and in particular how they transfer their rotational spindown energy into a relativistic wind.

W44 is one of the first remnants for which hard band $\mathrm{X}$-ray imaging was used to isolate a pulsar and its associated PWN from the brighter, softer thermal X-ray emission associated with the remnant's shock-heated gas. Neither the pulsar nor the nebula is apparent in low energy $\mathrm{X}$-ray images, such as that from the ROSAT PSPC (Rho et al. 1994). Using ASCA and its broader band imaging, however, Harrus et al. (1996) showed that while the PWN is invisible in the broad band image, it becomes the dominant feature above $4 \mathrm{keV}$. The centroid of the X-ray emission is consistent with the location of the pulsar, PSR B1853+01 (Wolszczan, Cordes \& Dewey 1991). ASCA's modest angular resolution precluded spatially distinguishing the synchrotron nebula from the surrounding diffuse emission, but Harrus et al. showed that the spectrum of the region including the PWN has a hard continuum component not detected elsewhere in W44. The techniques pioneered in Harrus et al. (1996) have subsequently been used to identify stellar remnants or synchrotron nebulae in other remnants (e.g., IC 443 - Keohane et al. 1997; MSH 15-56 - Plucinsky 1998; G292.0+1.8 - Torii, Tsunemi, \& Slane 1998). The most important consequence of this approach is the dramatic increase in the number of supernova remnants with identified synchrotron nebulae and/or compact stellar remnants.

The discovery of the X-ray synchrotron nebula occurred contemporaneously with the mapping of the pulsar wind nebula in the radio (Frail et al. 1996). At $1.4 \mathrm{GHz}$ it appears cometary in shape with an extent of $\sim 2.5$ arc minutes. The pulsar is located at the southern extremity. The radio surface brightness peaks at the widest part of the tail, approximately 1 arc minute north of the pul- sar. Frail et al. interpret this structure as the result of the pulsar's motion through the interior of the remnant. Using three independent techniques, they derive a velocity of the pulsar of approximately $375 \mathrm{~km} / \mathrm{s}$. The radio emission has a nonthermal spectrum with a spectral index of $0.12 \pm 0.04$, and it is $17 \pm 4$ percent polarized. The spectral index distinguishes the PWN from the surrounding emission associated with $W 44(\Gamma \sim$ 0.33 ); the spectrum and degree of polarization are similar to other pulsar wind nebulae. Using a combination of the X-ray and radio properties, Frail et al. estimate some key pulsar wind nebula parameters, including magnetic field strength, and the Lorentz, factor $\gamma$ of the electrons near the spectral break between the radio and X-ray slopes.

Giacani et al. (1997) presented radio and X-ray images of W44 as a whole. The PWN is apparent but inconspicuous in the radio. A line of $\mathrm{H} \alpha$ filaments lies along the eastern edge of the PWN, but there is no general correspondence with radio features, and it is unclear whether this emission is associated with the PWN or with shock heated material near the PWN only in projection.

The distance to W44 has generally been taken to be around $3 \mathrm{kpc}$, based on measurements of $\mathrm{H} \mathrm{I}$ absorption and $1720 \mathrm{MHz}$ maser lines (Caswell et al. 1975; Claussen et al. 1996). The analytical modeling of Cox et al. (1999) refines this distance to be between 2.5 and $2.6 \mathrm{kpc}$. We use a distance of $2.6 \mathrm{kpc}$, and scale parameters in terms of $\mathrm{d}_{2.6}$. The implications of using this refined value are minor. A luminosity estimate, for instance, is reduced by 25 percent, which is probably well within the uncertainty of the estimate.

\section{Observations and analysis}

W44 was observed using ACIS-S on 31 October, 2000 for $45.5 \mathrm{ks}$. As the primary goal of the observation was to understand the nature of the centrally peaked thermal emission in this mixedmorphology SNR, the S3 chip was pointed at the remnant center. The results of that investigation will be reported elsewhere. A fortuitous observation date made it possible to orient the spacecraft roll angle to place the pulsar squarely within the front-illuminated S2 chip. Although the calibration of S2 is not as complete as that of S3, it is adequate for the analysis we perform here. 
The data were analyzed using a combination of public an d custom software tools. We used CIAO tools version 2.2 and the calibrations available with the CIAO calibration database 2.9. An initial image of the pulsar nebula in the $2.0-8.0 \mathrm{keV}$ band was formed from the events file after point source removal, and smoothed with a 4."4 (9 ACIS pixel) HWHM Gaussian. Spectra were extracted using custom IDL software designed to extract counts within predefined brightness contours. Spectral fitting was performed using XSPEC v.11.1.0.

\section{Images}

In Figure 1 we show images of the region of W44 in the S2 field of view. The soft band (0.3$2.2 \mathrm{keV}$ ) image (Fig. 1a) is dominated by diffuse thermal emission from W44. Several unresolved sources are apparent; the pulsar is one of these, though not the brightest. The pulsar is located along the edge of an emission plateau stretching approximately southeast to northwest. It is the relatively low brightness of the pulsar and its fortuitous location along the ridge that rendered it undetectable to the ROSAT HRI, whose 0.2-2.4 $\mathrm{keV}$ band pass is similar to that of the soft band image displayed here. In contrast, the surface brightness of the thermal emission from W44 is substantially lower in the hard band $(2.2-8.2 \mathrm{keV})$ image (Fig. 1b). The unresolved sources are all still apparent; again, the pulsar is not the brightest. What makes the pulsar stand out is the enveloping diffuse emission, surrounding it and extending northward.

Using the hard image to avoid contamination by diffuse emission, we extracted the following position for PSR B1853+01: $18 \mathrm{~h} 56 \mathrm{~m} 10.653 \pm 0.028 \mathrm{~s}$; $01^{\circ} 13^{\prime} 21.3 \pm 0.36^{\prime \prime}$ (J2000). This can be compared with the radio position $18 \mathrm{~h} 56 \mathrm{~m} 10.8 \mathrm{~s} ; 01^{\circ} 13^{\prime} 28^{\prime \prime}$ (Frail et al. 1996). The origin of the $7^{\prime \prime}$ offset in declination is unknown. Frail et al. (1996) assign an error of $1^{\prime \prime} .5$ to the radio position. It is larger than the nominal $3^{\prime \prime} \mathrm{X}$-ray coordinate uncertainty from Chandra (Chandra Observatory Proposer's Guide), although the uncertainty for an outlying chip might be larger than this. Interestingly, when undertainties are taken into account, the X-ray position is consistent with the presumably less accurate position from radio timing reported by Wolszczan, Cordes \& Dewey (1991) at 18h 56m 10.9s; $01^{\circ} 13^{\prime} 20^{\prime \prime} .6$. It should be noted that the offset cannot be a result of proper motion, which has been estimated to be 25 mas/yr (Frail et al. 1996).

In Figure 2 we show a close up of the hard diffuse emission surrounding PSR B1853+01, and compare it with the radio map of Frail et al (1996). Both show diffuse emission, cometary in shape, trailing toward the north. The resemblance is close enough to suggest that this feature is the $\mathrm{X}$-ray PWN. Nevertheless, clear differences exist between the $\mathrm{X}$-ray and radio images. Unlike the radio emission, the $\mathrm{X}$-ray emission peaks at the pulsar. Additionally, the X-ray extent is about half the radio. The real $\mathrm{X}$-ray extent is slightly (about 20 percent) less than what is apparent in the figures. Due to the lack of counts, the image has been smoothed, thus increasing the apparent $\mathrm{X}$-ray extent.

An elliptical region of intermediate $\mathrm{X}$-ray surface brightness extends $1-2^{\prime}$ in all directions beyond the radio nebula, and a brighter ridge extends to the south and north. Our data lack sufficient signal to allow us to determine whether this emission is nonthermal, and thus associated with the PWN, or is thermal emission from W44.

\section{Spectra}

We have extracted spectra for the pulsar, the entire PWN region, and the two subregions indicated in Figure 2. Using the smoothed image to create image masks, we extracted spectra from the events file for regions of the pulsar nebula with surfce brightness between 3.3 and $5.7 \times 10^{-4}$ counts $\mathrm{s}^{-1} \operatorname{arcsec}^{-2}$, and surface brightness greater than $5.7 \times 10^{-4}$ counts $\mathrm{s}^{-1} \operatorname{arcsec}^{-2}$ (but excluding the pulsar itself). We used count-weighted response files (created with the CIAO routines mkwarf and $m k r m f$ ) to fit the spectra between 2.2 and $8.2 \mathrm{keV}$. We used XSPEC to map $\chi^{2}$ surfaces, and applied the Lampton, Margon, \& Bowyer (1976) criteria to find the $90 \%$ confidence interval for the value of the flux from the deabsorbed power-law models.

Great care has been exercised in fitting to remove the contribution of the thermal emission. Despite Chandra's ability to resolve spatially the PWN and to reduce the overall level of contamination due to thermal emission from $W 44$, there is still a substantial thermal contribution from the foreground and background thermal emission. 


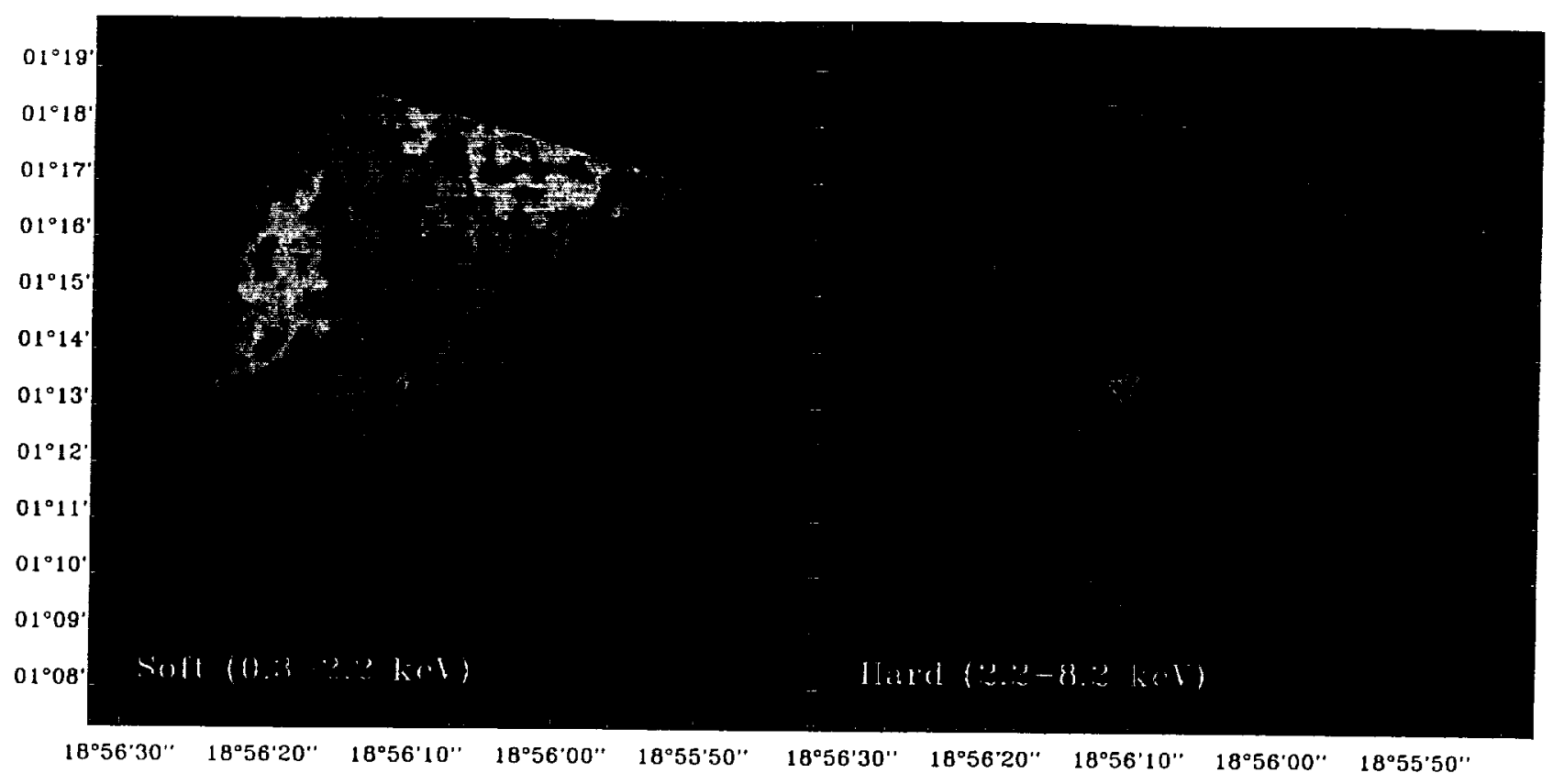

Fig. 1.- a) The soft (0.3-2.2 keV) broadband image from the ACIS-S2 chip. The black ellipses are the regions containing point sources which were removed before smoothing by a 4.4 HWHM Gaussian. The ellipses enclose $95 \%$ of the point source flux, and show the shape and orientation of the PSF. b) The hard $(2.2-8.2 \mathrm{keV})$ broadband image from the ACIS-S2 chip. The image has been smoothed by a $4^{\prime \prime} 4 \mathrm{HWHM}$ Gaussian.

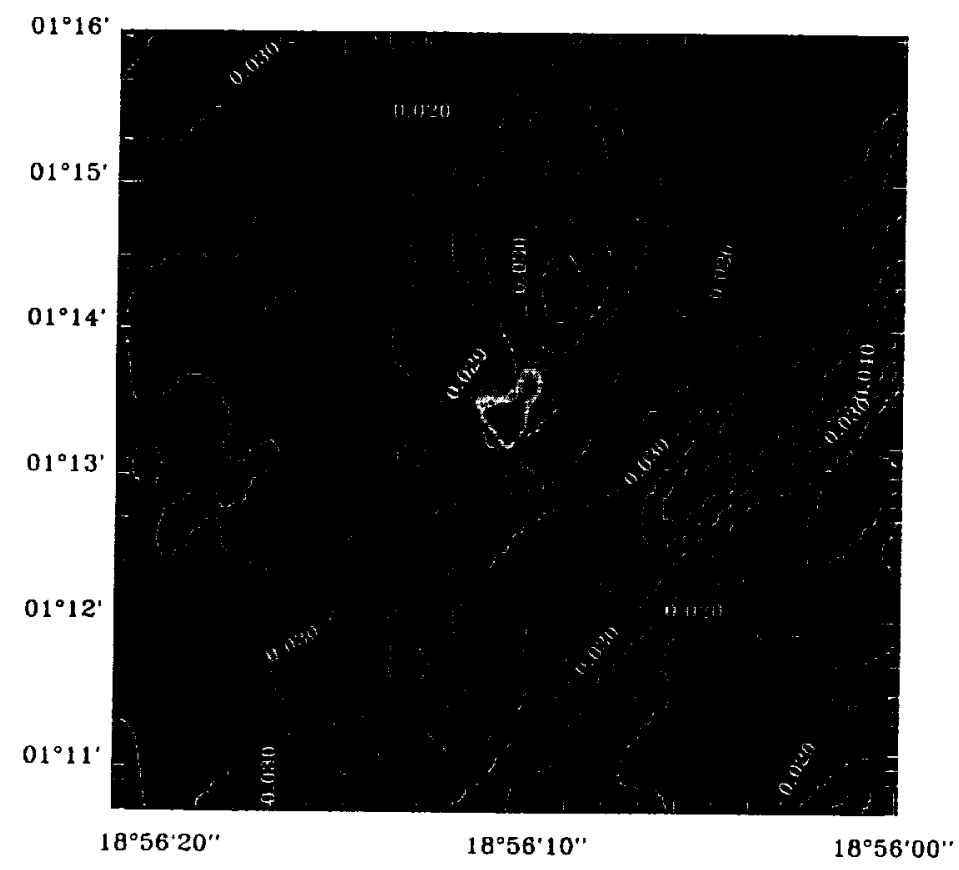

Fig. 2.- The region surrounding the PWN. Grayscale: The Chandra image in the 2.2-8.2 band smoothed with an 4"4 HWHM Gaussian. Contours: The $20 \mathrm{~cm}$ radio map from Frail et al. (1996), the units are $\mathrm{Jy} /$ beam, where the beam is $15^{\prime \prime} .8 \times 15^{\prime \prime} 4$. The dark contours are the boundaries of the subregions used for spectral fitting. The black ellipses indicate regions contaminated by point sources. 
During spectral fitting, we used several different fields drawn from nearby regions to represent the foreground and background thermal contribution. Incorporating these into the fits as a "background" file left insufficient signal for fitting the spectra below $2 \mathrm{keV}$. Thus all the fits were restricted to a band corresponding roughly to that of the hard band image in Fig. 1b. These restricted fits are insensitive to absorption by the column density of intervening material, which for W44 is on the order of $5 \times 10^{22} \mathrm{~cm}^{-2}$ (Rho et al. 1994). The fit results are insensitive to the specific choice of background field, largely as a result of the low overall signal-to-noise ratio of the spectra.

We show pertinent quantities from fits to absorbed power-law and absorbed Bremsstrahlung models in Table 1 . We restricted our fits to these simple models, given the small number of counts. All the models give formally acceptable fits, with $\chi_{\nu}^{2} \sim 1$. Although neither model is preferred for any of the spectra based on the quality of the fit, we interpret the spectrum in terms of the power law, the expected model for synchrotron emission.

The spectrum of PSR B1853+01 is hard, with a photon index $\Gamma=1.29 \pm 0.45$. There is no evidence for a second, softer, thermal component expected from the pulsar surface; the lower energy cut off of $2 \mathrm{keV}$ renders this component invisible. The overall spectrum of the extended nebula is considerably softer, with $\Gamma=2.2 \pm 0.2$. This value is similar to the $\Gamma \sim 2.3$ found by Harrus et al. (1996) for the spectrum of the pulsar plus nebula. This consistency is understandable given that the flux from the nebula is a factor of three higher than that from the pulsar. This value of $\Gamma$ is also similar to the average photon index of the Crab Nebula (2.2 - Willingale et al. 2001, and references therein).

In Figure 3 we show the best-fit photon index as a function of distance from the pulsar. The best-fit value of $\Gamma$ varies from 2.13 for inner region to 2.33 for the outer region, suggestive of spectral variation. The 90 percent confidence range for the spectral index from each region, however, contains the best-fit value of the other, rendering the search for spectral variation inconclusive.

\section{Discussion}

The ACIS observation of W44 has revealed the structure and spectrum of the PWN associated with the W44 pulsar. We find as in previous work (Harrus et al. 1996) that the pulsar and PWN stand out at energies above $\sim 2 \mathrm{keV}$. In the ACIS data they are detected for the first time at all energies down to the $\sim 1 \mathrm{keV}$ interstellar cutoff (but only because of our ability to discern the PWN's true shape from the hard band image). The PWN is clearly extended. It is also highly asymmetric, with significantly greater extent to the north, opposite the pulsar's apparent projected direction of motion. The spectra of the pulsar and PWN can be described as power laws. There is no evidence for a $\sim 10^{6} \mathrm{~K}$ thermal component that might be associated with surface emission from the pulsar. Such emission is presumably not detectable as a consequence of the background thermal emission from W44 and/or the high column density. The nebular spectrum is softer than the pulsar, and hints at steepening with distance from the pulsar.

The X-ray morphology of the PWN dif fers crucially from the radio morphology in two ways. First, it is a factor of two smaller in extent along the pulsar direction of motion. This difference can be ascribed to the overall softening of the electron spectrum as the higher energy electrons lose their energy more rapidly via synchrotron radiation. Second, the X-ray surface brightness peaks at or very near the pulsar, and decreases monotonically with distance from it. In contrast, the PWN has a more complex radio structure. The pulsar is embedded in a neck of emission $10^{\prime \prime}$ wide that connects with the larger cometary nebula $\sim 15^{\prime \prime}$ to the north. There is little diffuse radio emission surrounding the pulsar itself. A bridge of emission connects the pulsar and the PWN to its north. The radio surface brightness peaks not at the pulsar but near the northern edge of the Xray nebula. It is unusual to see such pronounced structural differences in a PWN. More usually the $\mathrm{X}$-ray and radio surface brightness profiles resemble each other and peak near the pulsar. It is possible that low surface brightness structure associated with the PWN in both the radio and the $\mathrm{X}$-ray is lost as a consequence of low contrast with the surrounding emission from the thermal remnant. 
TABLE 1

Fit Values for Pulsar and PWN Spectra

\begin{tabular}{lrrrrrrrr}
\hline Region & $\begin{array}{c}\text { Counts } \\
2-8 \mathrm{keV}\end{array}$ & $\begin{array}{c}\text { Area } \\
\text { pixels }\end{array}$ & Gamma & Red. $\chi^{2}$ & $\begin{array}{c}\text { Flux } \\
\text { erg cm }\end{array} \mathrm{s}^{-2} \mathrm{~s}^{-1}$ & $\mathrm{kT}$ & Red. $\chi^{2}$ & d.f \\
\hline PSR & 94 & 476 & $1.28 \pm 0.48$ & 0.68 & $5.96_{-1.40}^{+1.56} \times 10^{-14}$ & $55.0 \pm 230.0$ & 0.67 & 3 \\
Inner & 164 & 1821 & $2.12 \pm 0.40$ & 1.08 & $7.57_{-1.39}^{+1.45} \times 10^{-14}$ & $5.40 \pm 2.94$ & 1.16 & 7 \\
Both & 576 & 9707 & $2.20 \pm 0.22$ & 1.10 & $2.11_{-0.24}^{+0.25} \times 10^{-13}$ & $4.27 \pm 1.10$ & 1.11 & 30 \\
Outer & 412 & 7886 & $2.33 \pm 0.29$ & 1.12 & $1.38_{-0.20}^{+0.20} \times 10^{-13}$ & $3.65 \pm 1.05$ & 1.09 & 21 \\
Back & 1441 & 76335 & & & & & & \\
\hline
\end{tabular}

aPixels are 0!' $492 \times 0$ 0. 492, the "native" ACIS pixel size.

bUnabsorbed 2.2-8.2 keV band flux; calculated using the power-law model.

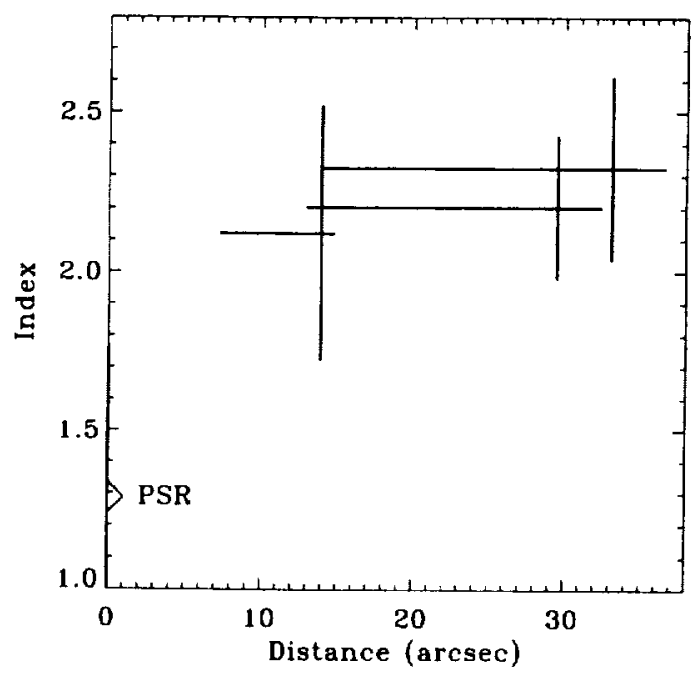

Fig. 3.- The photon index as a function of distance from the pulsar. The horizontal bars indicate the radii over which the index was measured. The vertical bars mark the pixel-weighted mean distance from the pulsar for each region. 
Is is surprising that any extent of the X-ray nebula is found. Based on synchrotron lifetime arguments, electrons sufficiently energetic to produce X-rays were expected only in close proximity to the pulsar (Harrus et al. 1996). In principle, the combination of the $\mathrm{X}$-ray and radio profiles along the direction of the pulsar motion provides a record of the history of the production of energetic particles in the pulsar magnetosphere.

In Table 2, we compare the values of the various parameters for the W44 PWN with those of other PWNs, including the young Crab and $3 \mathrm{C} 58$, the intermediate age G21.5-0.9, and the older IC 443 PWN. In the discussion that follows, we describe how the values for the W44 parameters were inferred, and compare them with the values from the other objects.

The X-ray flux in the $2.2-8.2 \mathrm{keV}$ band, where the spectrum was measured, is $2.7 \times 10^{-13} \mathrm{ergs} \mathrm{cm}^{-2} \mathrm{~s}^{-1}$. This is considerably lower than that inferred by Harrus et al. (1996), but it can be expected that the considerably greater difficulty of extracting the PWN signal from the lower angular resolution ASCA data led to a less accurate flux estimate. (Note that the ASCA detection is barely significant.) Extrapolation of the unabsorbed flux to the Einstein band $(0.2-4.0 \mathrm{keV})$ yields a value of $7 \times 10^{-13}$ ergs $\mathrm{cm}^{-2} \mathrm{~s}^{-1}$, and a corresponding luminosity of $6 \times 10^{32} \mathrm{~d}_{2.6}{ }^{2} \mathrm{ergs} \mathrm{s}^{-1}(40,000$ times less luminous than the $\mathrm{Crab}$ ). This may be compared with the luminosity of $8 \times 10^{32} \mathrm{ergs} \mathrm{s}^{-1}$ predicted using the empirically derived relation for pulsar wind nebulae (Seward \& Wang 1988) $\log \left(\mathrm{L}_{x}\right)=1.39 \log (\dot{\mathrm{E}})-16.6$, where $\mathrm{L}_{x}$ is the PWN $\mathrm{X}$-ray luminosity and $\dot{\mathrm{E}}$ is the rate of rotational energy loss by the pulsar, which has a value of $4.3 \times 10^{35} \mathrm{erg} \mathrm{s}^{-1}$ (Wolszczan, Cordes \& Dewey 1991). The correspondence is remarkable, considering the nebula's atypical morphology and history.

The spectral index is not observed to change radically with distance from the pulsar, in contrast to all the other PWNs listed in Table 2. Most interesting is the contrast with the most similar object known, the PWN in IC 443, whose spectral index varies by 0.7 (Bocchino \& Bychkov 2001). As pointed out above, the overall nebular X-ray spectral index is also similar to that of the Crab Nebula. Chevalier (2000) developed a model for the Xray luminosity of PWNs in which he claimed that the X-ray spectral index is an indicator of the efficiency with which the particle energy is converted into X-ray emission. In particular, he argues that in PWNs with Crab-like X-ray (and thus electron) spectra, the X-ray luminosity should be produced with high efficiency. For simplicity, his model assumes a constant magnetic field. The field in the W44 PWN might be nearly constant, given the lack of X-ray spectral index variation. According to this model, the value and constancy of the $\mathrm{X}$-ray spectral index suggest that the W44 PWN should have an $\dot{\mathrm{E}} / \mathrm{L}_{x}$ that is much closer to the Crab than the observed factor-of-five difference. Since the only other PWN that seems Crab-like in this regard according to Chevalier (the LMC remnant 0540-69.3) is, like the Crab, about 1,000 $\mathrm{yr}$ old, perhaps some additional factor, such as the age of the nebula, needs to be incorporated in the model.

In the widely accepted model of PWNs, the energy source is the spindown energy of the pulsar. The energy is efficiently transferred into a relativistic wind with some characteristic Lorentz factor $\gamma$. This energy is divided between Poynting (magnetic and electric) flux and particle energy flux, the ratio of which is the parameter $\sigma$. The initial interaction between the wind and the surrounding medium forms a termination shock, usually creating wisps and filaments identifiable in the radio and visible bands (and now in the $\mathrm{X}$-ray, using Chandra). In the pulsar's magnetosphere, where the particles are created by pair production, the wind is expected to be magnetically dominated $(\sigma \geq 1)$. By the time the wind reaches the termination shock, observations indicate it becomes particle dominated $(\sigma<1)$. No clear theoretical explanation for this transition has emerged (Arons 1998). Measurements of wind parameters in other PWNs typically show $\sigma$ on the order of a few times $10^{-3}$ (see Table 2). The composition of the particle flux is also of interest. This is characterized by the parameter $k$, the ratio between the energy density in electrons, and that in all other particles (positrons, protons and other nuclei). While models commonly assume an electron-positron plasma with $\mathrm{k}=1$, Frail et al. (1996) have shown that in the W44 wind nebula $5 \leq \mathrm{k} \leq 30$.

The Chandra observation allows us to refine estimates of these parameters characterizing the $\mathrm{PWN}$, as well as the magnetic field strength $\mathrm{B}$. Un- 
TABLE 2

Parameters for Various Pulsar Wind Nebulae

\begin{tabular}{|c|c|c|c|c|c|}
\hline & $3 \mathrm{C} 58$ & Crab & G21.5-0.9 & W44 & IC 443 \\
\hline $\mathrm{X}$-ray Size (pc) & $10 \times 6$ & 1.2 & 7 & $1 \times 0.5$ & $3.5 \times 2$ \\
\hline Radio Size (pc) & $10 \times 6$ & $3.5 \times 2.3$ & $2.2 \times 1.3$ & $2 \times 1$ & $1.3 \times 0.9$ \\
\hline Distance (kpc) & 3.2 & 2 & 5 & 2.6 & 1.5 \\
\hline Age (yr) & 820 & 950 & $3-6000$ & 20,000 & 30,000 \\
\hline $\mathrm{L}_{x}\left(\right.$ ergs s$\left.{ }^{-1}\right)$ & $2.4 \times 10^{34}$ & $2.1 \times 10^{37}$ & $3.3 \times 10^{35}$ & $6.0 \times 10^{32}$ & $2.6 \times 10^{33}$ \\
\hline$\dot{\mathrm{E}}\left(\right.$ ergs s $\mathrm{s}^{-1}$ ) & $4.0 \times 10^{36}$ & $4.7 \times 10^{38}$ & $3-6 \times 10^{37}$ & $4.3 \times 10^{35}$ & $1.3 \times 10^{36}$ \\
\hline $\mathrm{L}_{x} / \dot{\mathrm{E}}$ & 0.006 & 0.05 & $0.005-0.01$ & 0.001 & 0.002 \\
\hline$\Gamma$ range & $1.85,2.4$ & $1.6-2.3$ & $1.5-2.8$ & $2.1-2.3$ & $1.6-2.3$ \\
\hline$\dot{E}\left(\right.$ ergs s $\left.^{-1}\right)$ & $4.0 \times 10^{36}$ & $4.7 \times 10^{38}$ & $3-6 \times 10^{37}$ & $4.3 \times 10^{35}$ & $1.3 \times 10^{36}$ \\
\hline & $2-15 \times 10^{-3}$ & $3 \times 10^{-3}$ & $0.4-1.1 \times 10^{-3}$ & $0.4-1.0 \times 10^{-3}$ & \\
\hline Cutoff frequency $(\mathrm{Hz})$ & $5 \times 10^{10}$ & $1 \times 10^{13}$ & & $8 \times 10^{12}$ & $1 \times 10^{11}$ \\
\hline References $^{\mathrm{a}}$ & 1,2 & 3,4 & 5 & 6,7 & 8 \\
\hline
\end{tabular}

a REFERENCES - (1) Torii et al. 2000; (2) Bocchino et al. 2001; (3) Kennel \& Coroniti 1984a; (4) Willingale et al. 2001; (5) Safi-Harb et al. 2001; (6) Wolszczan, Cordes \& Dewey 1991; (7) Frail et al. 1996; (8) Bocchino \& Bychkov 2001.

der the assumption that the break between the radio and the X-ray spectra arises from synchrotron losses, Frail et al. (1996) have shown how an estimate of the turnover frequency, $\nu_{B}$, of the PWN spectrum leads to estimates of the nebular magnetic field strength and the Lorentz factor, $\gamma$, for the electrons near $\nu_{B}$. From the improved measurement of the X-ray flux and spectral index of the PWN, we find a best-fit cutoff frequency $\nu_{B}=$ $8 \times 10^{12} \mathrm{~Hz}$. The reduced flux value is the primary reason why the estimate of the synchrotron cutoff in the present work is substantially lower than that of Harrus et al. (1996). Our best-fit $\nu_{B}$ is similar to the Crab's break frequency of $10^{13} \mathrm{~Hz}$. Using the equations reproduced by Frail et al. from $\mathrm{Pa}$ cholczyk (1970) we then find:

$\mathrm{B}=1040 \mu \mathrm{G} \quad\left(\nu_{B} / 10^{12} \mathrm{~Hz}\right)^{-1 / 3}\left(\mathrm{t}_{\text {res }} / 1000\right.$ $\mathrm{yr})^{-2 / 3}=160 \mu \mathrm{G}$; and

$$
\gamma \sim 10^{5}\left(\nu_{B} / 10^{12} \mathrm{~Hz}\right)^{1 / 2}(\mathrm{~B} / 100 \mu \mathrm{G})^{-1 / 2}=
$$
$2.2 \times 10^{5}$

Here $t_{r e s}$ represents the age of the nebula, which Frail et al. take to be $5,700 \mathrm{yr}$ based on the nebular extent in the radio.

The new cutoff frequency estimate falls squarely between the values used by Frail et al. (1996) to bound the value of $\mathrm{k}$. Using our best value of $\nu_{B}$, we find $\mathrm{k} \sim 10$, consistent with their conclusionthat the particle energy is electron dominated.
We can estimate the value of the magnetization parameter $\sigma$, following the approach used by Torii et al. (2000) for $3 \mathrm{C} 58$. They used the formalism developed by Kennel and Coroniti $(1984 a, b)$ for the Crab, who showed that $\sigma$ is related to the velocity profile of the nebula by $\mathrm{v}(\mathrm{z}) / \mathrm{c} \sim 3 \sigma\left[1+\left(3 \mathrm{z}^{2}\right)^{-1 / 3}\right]$. Here $\mathrm{z}=\mathrm{r} / \mathrm{r}_{s}$, the ratio between radius and the distance between the pulsar and the termination shock. Torii et al. estimated $\sigma$ by es timating $\mathrm{r}_{N}$ and $\mathrm{v}_{N}$, the values at the edge of the radio nebula. Unlike $3 \mathrm{C} 58$, in which the pulsar shows little proper motion, care must be exercised here in estimating global nebular parameters like size in the presence of substantial pulsar motion.

For the nebular size $r_{N}$ we take the largest dimension in the radio nebula perpendicular to the direction of motion, which is 1 arc minute or $0.75 \mathrm{~d}_{2.6} \mathrm{pc}$. For the nebular age, we cannot use the nominal spin down age of 20,000 yr. Given the estimated proper motion, the pulsar has moved $\sim 8$ arc minutes, an angular distance far larger than the size of the observed nebula. Instead we use the age of the radio nebula, $5,700 \mathrm{yr}$, which is based on synchrotron lifetime arguments (Frail et al. 1996). Assuming homologous expansion, we find $\mathrm{v}_{N}$ (= $2 / 5 \times 0.75 \mathrm{pc} / 5,700 \mathrm{yr}) \sim 50 \mathrm{~km} \mathrm{~s}^{-1}$; assuming constant expansion, we find $\mathrm{v}_{N} \sim 130 \mathrm{~km} \mathrm{~s}^{-1}$. Another estimate of the nebular lifetime can be de- 
rived from the time required by the pulsar to move from the location corresponding to the largest perpendicular dimension to its current location; 1.5 arc minutes divided by the proper angular motion of $25 \mathrm{mas} / \mathrm{yr}$, or $3,600 \mathrm{yr}$. This simple calculation suggests that the lifetime used can be off by not more than a factor of $\sim 1.6$.

There is no evidence in the visible, radio or $\mathrm{X}$ rays of the wisps observed in the Crab or $3 \mathrm{C} 58$ that are interpreted as the termination shock. Since the termination shock represents the location where the ram pressure of the relativistic wind from the pulsar $(\dot{\mathrm{E}} /(4 \pi \mathrm{cr}))$ equals the pressure in the nebula (presumably dominated by magnetic pressure), we can estimate $r_{s}$ by equating these two quantities at the termination shock. Thus $\dot{\mathrm{E}} /\left(4 \pi \mathrm{c} \mathrm{r}_{s}\right)=1 / 3\left(\mathrm{~B}^{2} /(8 \pi)\right)$. For $\mathrm{B}=160 \mu \mathrm{G}$ and $\dot{\mathrm{E}}=4.3 \times 10^{35} \mathrm{ergs} \mathrm{s}^{-1}$ (Wolszczan, Cordes \& Dewey 1991), we find $\mathrm{r}_{s} \sim 0.02 \mathrm{pc}$, leading to a value of $\mathrm{z}_{N}=\mathrm{r}_{N} / \mathrm{r}_{s} \sim 80$, which can be compared with $z_{N}$ of 20 for the much younger and more energetic Crab, and 15-100 for 3C 58 (Torii et al. 2000).

Combining the estimates for $\mathrm{z}_{N}$ and $\mathrm{v}_{N}$, we estimate $\sigma \sim 0.4-1.0 \times 10^{-3}$, depending upon which value of $v_{N}$ is assumed. As indicated in Table 2 , this value of $\sigma$ is lower than that found for the younger PWNs, including the Crab $\left(3 \times 10^{-3}\right.$

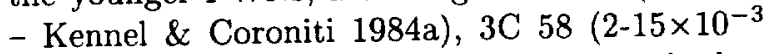
- Torii et al. 2000), but is consistent with that for the older G21.5-0.9 (4-11 $\times 10^{-4}$ Safi-Harb et al. 2001). While the sample of measurements is small, it suggests that $\sigma$ may decrease with PWN age, but it either stays near a value of $\sim 10^{-3}$ over many thousands of years or $\sigma$ must have a value around $10^{-3}$ if a PWN is to be observable.

We can use the refined magnetic field estimate to obtain a synchrotron lifetime estimate for the hard band X-ray emission pictured in Figs. 1 and 2. For a magnetic field strength $B$ in Gauss, and a photon frequency $\nu$ in $\mathrm{Hz}$, the synchrotron lifetime $\tau$ in seconds is $\sim 6 \times 10^{11} \mathrm{~B}^{-3 / 2} \nu^{-1 / 2}$. For the estimated value of $\mathrm{B}(160 \mu \mathrm{G})$ and a photon frequency of $5 \times 10^{17} \mathrm{~Hz}(\sim 2.5 \mathrm{keV})$, we find $\tau \sim 3 \times 10^{8} \mathrm{~s}$, or approximately $15 \mathrm{yr}$. This lifetime is very short compared with the $\sim 20,000 \mathrm{yr}$ pulsar age (Wolszczan, Cordes \& Dewey 1991) or the apparent nebular age of $5,700 \mathrm{yr}$. The short lifetime, along with the $\sim 1 \mathrm{~d}_{3}$ pc extent of the $\mathrm{X}$-ray nebula implies that the $\mathrm{X}$-ray emitting elec- trons $\left(\nu \geq 5 \times 10^{17} \mathrm{~Hz}\right)$ have a high streaming velocity, $\sim 1 / 3 \mathrm{c}$. The high streaming velocity in turn suggests that the magnetic field in the extended nebula is ordered, and oriented along the wake of the pulsar's motion. It is reasonable to expect an ordered field if one considers that the PWN magnetic field should be considerably stronger than any residual magnetic field in the remnant interior, even in the mixed-morphology W44 whose interior density is substantially higher than a typical shell-like SNR.

As a final note, one feature of potential future interest is the possible low surface brightness extended $\mathrm{X}$-ray emission. If it is nonthermal emission associated with the PWN, then W44 would be the third PWN with a larger apparent extent in the X-ray than in the radio. Similar structures have been found in the plerionic remnant G21.5-0.9 (Slane et al. 2000; Warwick et al. 2001) and the PWN inside IC 443 (Bocchino \& Bychkov 2001), with clearly nonthermal X-ray emission extending well beyond the radio nebula. If the emission in W44 is confirmed, it is possible that low surface brightness radio emission is also produced but is invisible against the foreground emission from the shocked gas in the supernova remnant. The existence of such low level emission has been speculated upon by Warwick et al. (2001) for G21.5-0.9, where it might be easier to detect. The reality of the $\mathrm{X}$-ray emission should be straightforwardly demonstrated by XMM/Newton, whose substantially higher throughput will facilitate more accurate measurements of the PWN spectrum and its variations.

In summary, the ACIS observation of the pulsar wind nebula surrounding PSR B1853+01 in W44 reveals an extended nebula, half the size of the radio $\mathrm{PWN}$. Spectroscopy reveals a significant difference between the power law photon index of PSR B1853+01 $(\Gamma \sim 1.4)$ and that of the nebula $(\Gamma \sim 2.2)$. Variation of the photon index within the nebula has not been detected. The $\mathrm{X}$-ray size and spectrum of the PWN have allowed us to estimate key parameters, including magnetic field strength, the average $\gamma$ of the particles in the wind, the magnetization parameter $(\sigma)$, and the ratio $\mathrm{k}$ of electrons to other particles. We find that despite the unusual morphology produced by the high velocity and age of the pulsar, the W44 PWN has properties similar typical of 
other PWN. A number of unresolved issues, such as the possible existence an extended nebula and spectral variation within the nebula, make this fascinating object deserving of more extensive study. It is hoped that deeper observations using either Chandra or XMM/Newton can provide more exact values of the observables and facilitate measurements of their variation with distance from PSR B1853+01, leading in turn to more robust estimates of the PWN parameters.

We acknowledge the tireless efforts of the CXC staff, and the team that developed the Chandra observatory. We would like to thank D. Frail for use of the $20 \mathrm{~cm}$ map, and $\mathrm{K}$. Arnaud with assistance while we were developing the IDL software used to extract irregular regions. Support for this work for R.L.S. and K.D.K. was provided by the National Aeronautics and Space Administration through grant G01-2057A issued by the Chandra $\mathrm{X}$-ray Observatory for and on behalf of NASA under contract NAS8-39073.

\section{REFERENCES}

Arons, J. 1998, Mem. S. A. It., 69, 989

Bocchino, F., \& Bychkov, A.M. 2001, A \& A, 376, 248

Bocchino, F., Warwick, R.S., Marty, P., Lumb., D., Becker, W., \& Pigot, C. 2001, A\&A, 369, 1078

Cox, D.P., Shelton, R.L., Maciejewski, W., Smith, R.K., Plewa, T., Pawl, A., \& Rozyczka, M. 1999, ApJ, 524, 179

Caswell, J.L., Murray, J.D., Roger, R.S., Cole, D.J., \& Cooke, D.J. 1975, A\&A, 45, 239

Claussen, M.J., Frail, D.A., Goss, W.M., \& Gaume, R.A. 1997, ApJ, 489, 143

Chevalier, R.A. 2000, ApJ, 539, L45

Frail, D.A., Giacani, E.B., Goss, W.M., \& Dubner, G. 1996, ApJ, 464, L165

Giacani, E.B., Dubner, G.M., Kassim, N.E., Frail, D.A., Goss W.M., Winkler, P.F., \& Williams, B.F. 1997, AJ, 113, 1379

Harrus, I.M., Hughes, J.P., \& Helfand, D.J. 1996, ApJ, $464, \mathrm{~L} 161$

Kennel, C.F., \& Coroniti, F.V. 1984a, ApJ, 283, 694

Kennel, C.F., \& Coroniti, F.V. 1984b, ApJ, 283, 710

Keohane,J.W., Petre, R., Gotthelf, E.V., Ozaki, M., \& Koyama, K. 1997, ApJ, 48 4, 350
Lampton, M., Margon, B., \& Bowyer, S. 1976, ApJ 208, L177

Pacholczyk, A.G. 1970, Radio Astrophysics (San Francisco: Freeman)

Plucinsky, P.P. 1998, Mem.S.A.It., 69, 939

Rho, J.-H., Petre, R., Schlegel, E.M., \& Hester, J.J. 1994, ApJ, 430, 757

Seward, F.D., \& Wang, Z.R. 1988, ApJ, 332, 199

Slane, P., Chen, Y., Schulz, N.S., Seward, F.D., Hughes, J.P., \& Gaensler, B.M. 2000, ApJ, 533, L29

Torii, K., Tsunemi, H., \& Slane, P. 1998, in IAU Symp. 188, The Hot Universe, ed. K. Koyama, K. Kitamoto, \& M. Itoh (Dordrecht: Kluwer), 258

Torii, K., Slane, P., Kinugasa, K., Hashimotodani, K., \& Tsunemi, H. 2000, PASJ, 52, 875

Warwick, R.S., et al. 2001, A\&A, 365, L248

Willingale, R., Aschenbach, B., Griffiths, R.G., Sembay, S., Warwick, R.S., Becker, W., Abbey, A.F., \& Bonnet-Bidaud, J.M. 2001, A\&A, 365, L212

Wolszczan, A., Cordes, J.M., \& Dewey, R.J. 1991, ApJ, 372, L99

This 2-column preprint was prepared with the AAS IATEX macros $\mathrm{v} 5.0$. 